\title{
A class of concave Young functions possessing a positive fixed point
}

\author{
N. K. Agbeko
}




\title{
A CLASS OF CONCAVE YOUNG FUNCTIONS POSSESSING A POSITIVE FIXED POINT
}

\author{
N. K. AGBEKO \\ Received 10 June, 2008
}

Abstract. We obtained the class of all concave Young functions which possess a positive fixed point.

2000 Mathematics Subject Classification: 47H10, 37C25, 47H25

Keywords: concave Young functions, degree of contraction, fixed points

\section{INTRODUCTION}

Let $\varphi:(0, \infty) \rightarrow(0, \infty)$ be a right-continuous and decreasing function such that it is integrable on every finite interval $(0, x)$. It is easily seen that the function $\Phi$ : $[0, \infty) \rightarrow[0, \infty)$, defined by the equality

$$
\Phi(x)=\int_{0}^{x} \varphi(t) d t
$$

is a nonnegative, increasing and concave function with $\Phi(0)=0$. We further assume that $\Phi(\infty)=\infty$. Function $\Phi$ is thus referred to as a concave Young function in the literature, and the set of all such functions will be denoted by $y_{\text {conc }}$. For more about these functions see, e. g, [1-3,5].

In [3], we obtained the following results.

Proposition 1.1. Let $\Phi \in y_{\text {conc }}$ and $s \in(0, \infty)$ be arbitrary. Then

$$
|\Phi(x)-\Phi(y)| \leq \varphi(s)|x-y|
$$

for all numbers $x, y \in(s, \infty)$.

We sought for all those positive numbers that can be a fixed point for a given concave Young function.

Theorem 1.1. Let $\Phi \in y_{\text {conc }}$ and $c^{*}$ be any positive number. In order that the equality $\Phi\left(c^{*}\right)=c^{*}$ hold, it is necessary and sufficient that the range of the function $\left.\Phi\right|_{\left[c^{*}, \infty\right)}:\left[c^{*}, \infty\right) \rightarrow[0, \infty)$, defined by $\left.\Phi\right|_{\left[c^{*}, \infty\right)}(x)=\Phi(x)$, should equal the interval $\left[c^{*}, \infty\right)$. 
Let $\Phi \in y_{\text {conc }}$ with

$$
\int_{1}^{\infty} \frac{\varphi(t)}{t} d t<\infty .
$$

In [3], a number $c \in(0, \infty)$ was called the degree of contraction of $\Phi$ if

$$
\int_{c}^{\infty} \frac{\varphi(t)}{t} d t=1
$$

and

$$
\int_{c}^{b c} \frac{\varphi(t)}{t} d t=\varphi(c)
$$

for some $b \in(1, \infty)$. We intend to extend this notion to other concave Young functions which do not possess property (1.2).

\section{MAIN RESUlT}

Theorem 2.1. Let $\Phi \in y_{\text {conc }}$ be arbitrary with $\varphi$ denoting its derivative. In order that there be a constant $s>0$ for which $\varphi(s)<1$, it is necessary and sufficient that $\Phi$ admit a positive fixed point, i. e., $\Phi(x)=x$ for some number $x>0$.

Proof. To prove the sufficiency, assume that there is a number $s>0$ such that $\varphi(s)<1$. Then by recalling Proposition 1.1 one can easily observe that $\Phi$ is a contraction in the interval $(s, \infty)$. Consequently, the Contraction Principle [6] yields $\Phi(x)=x$ for some $x \geq s$. Next, let us show the necessity. Assume that there exists some $x_{0}>0$ for which $\Phi\left(x_{0}\right)=x_{0}$, but in the contrary $\varphi(t) \geq 1$ for all $t>0$. Then it is easy to check that $\Phi(x) \geq x$ for all $x>0$. Since $\Phi$ is a strictly concave and increasing function, the graph of $\Phi$ must lie below that of the line $y=x$ on the interval $\left(x_{0}, \infty\right)$. This fact, however, contradicts the inequality $\Phi(x) \geq x$ for all $x>0$.

Proposition 2.1. Let $\Phi \in y_{\text {conc }}$ be arbitrary with $\varphi$ denoting its derivative. If $x_{0} \in(0, \infty)$ is such that $\Phi\left(x_{0}\right)=x_{0}$, then $\varphi\left(x_{0}\right)<1$.

Proof. It is not difficult to see that $\Phi(t) \geq t \varphi(t)$ whenever $t \in(0, \infty)$. Assume the existence of some $x_{0} \in(0, \infty)$ for which $\Phi\left(x_{0}\right)=x_{0}$. Then, as noted above,

$$
x_{0}=\Phi\left(x_{0}\right) \geq x_{0} \varphi\left(x_{0}\right),
$$

and hence $\varphi\left(x_{0}\right) \leq 1$. Now, suppose that $\varphi\left(x_{0}\right)=1$. Since $\varphi$ is a decreasing function on $(0, \infty)$, there must be some $\varepsilon \in(0,1)$ such that $\varphi\left(x_{0}+\varepsilon\right)<1$, making $\Phi$ be a contraction on $\left(x_{0}+\varepsilon, \infty\right)$, via Proposition 1.1. But then it would mean that there must be some $x^{*} \in\left(x_{0}+\varepsilon, \infty\right)$ with $\Phi\left(x^{*}\right)=x^{*}$. Necessarily, it would ensue that $\Phi$ is not a concave function on the interval $\left(x_{0}, x^{*}\right]$, a contradiction. Therefore, $\varphi\left(x_{0}\right)<1$.

Now, we are in a position to reformulate the definition of the degree of contraction to cover a broader class of concave Young functions. 
Definition 2.1. A number $s>0$ is called the degree of contraction of a function $\Phi \in y_{\text {conc }}$ if $\varphi(s)=1$, where $\varphi$ is the derivative of $\Phi$.

We note in this case that $\varphi(s+\delta)<1$ for any positive number $\delta$, which makes $\Phi$ be a contraction on the interval $(s+\delta, \infty)$ for some suitable $\delta$. 3.

Example 1. The degree of contraction of $\Phi(x)=4 \sqrt{x+1}-4, x \in[0, \infty)$, equals

Example 2. For any fixed number $p \in(0,1)$, the degree of contraction of the function $\Phi_{p}(x)=x^{p}, x \in[0, \infty)$ is equal to $p^{1 /(1-p)}$.

Example 3. The function $\Phi(x)=\log (x+1), x \in[0, \infty)$, has no degree of contraction.

Example 4. The degree of contraction of function $\Phi(x)=2 \log (x+1)$ exists and equals 1 .

Example 5. The concave Young function $\Phi$ defined by $\Phi(x)=\frac{x}{2}+\sqrt{x}$ does not meet condition (1.2). Yet its degree of contraction exists and equals 1.

An algorithm for finding positive fixed points for concave Young functions:

Step 1: Input $\Phi(x)$ a concave Young function, $c_{0}$ a positive number.

Step 2: Compute the derivative $\varphi(x)$ of $\Phi(x)$.

Step 3: Starting from $c_{0}$ find an approximate root of the equation $\varphi(x)-1=0$ and put the result into $c$.

Step 4: If $c=0$ then STOP else GOTO Step 5.

Step 5: Starting from $c$ apply the Fixed Point algorithm, i.e.,

$x_{0}:=c ; x_{k+1}:=\Phi\left(x_{k}\right) ; k=k+1$.

\section{CONCLUDING REMARKS}

In dynamic models, stationary equilibrium is typically described as a solution of the equation $x=f(x)$, where $f$ is a mapping which determines the current state as a function of the previous state, or as a function of the expected future state. In many cases $x$ is a finite dimensional vector, and in general positive solutions (i. e., fixed points of $f$ ) are rather sought for. Problems of this kind have been investigated for decades, and often for concave functions. Alfred Tarski in [7] obtained, in particular, the following result.

Theorem 3.1 (Tarski). Suppose $f$ is an increasing function from $\mathbb{R}^{n}$ to $\mathbb{R}^{n}$ such that $f(a)>$ a for some positive vector $a$, and $f(b)<b$ for some vector $b>a$. Then $f$ has a positive fixed point.

For the proof we refer the reader, e. g., to [4]. In [4], J. Kennan obtained the result stated below by using Tarski's theorem and [4, Theorem 3.1]. He observed that it gave simple sufficient conditions for the existence and uniqueness of a positive fixed point. 
Theorem 3.2 ([4, Theorem 3.3]). Suppose that $f$ is an increasing and stricly concave function from $\mathbb{R}^{n}$ to $\mathbb{R}^{n}$ such that $f(0) \geq 0, f(a)>$ a for some positive vector $a$, and $f(b)<b$ for some vector $b>a$. Then $f$ has a unique positive fixed point.

We note that the concavity and increasing property of $f$ mean that every component $f_{k}(k=1, \ldots, n)$ of $f$, considered as a function from $\mathbb{R}^{n}$ to $\mathbb{R}$, is increasing and strictly concave in every argument $x_{j} \in \mathbb{R}, j=1, \ldots, n$.

\section{ACKNOWLEDGEMENT}

The author would like to thank Prof. Attila Házy for his valuable comments.

\section{REFERENCES}

[1] N. K. Agbeko, "Concave function inequalities for sub- (super-) martingales," Ann. Univ. Sci. Budapest. Eötvös Sect. Math., vol. 29, pp. 9-17 (1987), 1986.

[2] N. K. Agbeko, "Necessary and sufficient condition for the maximal inequality of concave Youngfunctions," Ann. Univ. Sci. Budapest. Eötvös Sect. Math., vol. 32, pp. 267-270 (1990), 1989.

[3] N. K. Agbeko, "Studies on concave Young-functions," Miskolc Math. Notes, vol. 6, no. 1, pp. 3-18, 2005.

[4] J. Kennan, "Uniqueness of positive fixed points for increasing concave functions on $\mathbb{R}^{n}$ : An elementary result," Review of Economic Dynamics, vol. 4, no. 4, pp. 893-899, 2001.

[5] J. Mogyoródi, "On a concave function inequality for martingales," Ann. Univ. Sci. Budapest. Eötvös Sect. Math., vol. 24, pp. 265-271, 1981.

[6] M. C. Reed, Fundamental ideas of analysis. New York: John Wiley \& Sons Inc., 1998.

[7] A. Tarski, "A lattice-theoretical fixpoint theorem and its applications," Pacific J. Math., vol. 5, pp. 285-309, 1955.

Author's address

\section{N. K. Agbeko}

Institute of Mathematics, University of Miskolc, H-3515 Miskolc-Egyetemváros, Hungary

E-mail address: matagbek@uni-miskolc.hu 